\title{
Vial-to-Pen: Community-Based Pharmacists Converting Insulin Regimens
}

Ashley N. Hannings, PharmD, BCACP ${ }^{1}$; Natasha M. Michaels, PharmD, BCACP²; Debbie Hiller, PharmD ${ }^{3}$, Macary Weck Marciniak, PharmD, BCACP, BCPS, FAPhA ${ }^{4}$; Stefanie P. Ferreri, PharmD, CDE, BCACP, FAPhA 4

${ }^{1}$ University of Georgia College of Pharmacy; ${ }^{2}$ Sona Pharmacy + Clinic, Asheville, North Carolina; ${ }^{3}$ BlueCross BlueShield of North Carolina, Durham, North Carolina; ${ }^{4}$ UNC Eshelman School of Pharmacy, University of North Carolina at Chapel Hill

\begin{abstract}
Objective: To describe a vial-to-pen conversion program driven by community-based pharmacists and determine conversion success rate.

Methods: A report based on prescription claims was generated and identified 200 prescriptions filled for an insulin vial product and syringes. Patients were contacted by community-based pharmacists during a five-month period and were informed of the availability and potential benefits of insulin pen delivery systems (IPDS). If the patient agreed to the switch, the pharmacist contacted the prescriber to obtain a new prescription. Prescription refill records were tracked for six months post-intervention to determine whether patients who were converted remained on the IPDS.

Results: The overall vial-to-pen conversion success rate was $26 \%$ out of 121 potential conversions. In addition, $52 \%$ of patients reached were willing to switch and prescribers approved $71 \%$ of the recommendations to switch from vial-to-pen. Of the prescriptions successfully converted to an IPDS, $84 \%$ of prescriptions were still dispensed as pen products six months following the conversion.

Conclusion: Community-based pharmacists can serve as a resource to provide education on insulin delivery options and increase use of IPDS in patients with diabetes.
\end{abstract}

Keywords: community pharmacy, insulin administration, insulin pen delivery systems, insulin vial, and insulin syringe

\section{Introduction}

Insulin is the most effective antihyperglycemic agent; however, only $29 \%$ of adults with diabetes use insulin for diabetes management. ${ }^{1}$ Many patients have concerns about its use, including risk of hypoglycemia, pain related to injection, social stigma, and difficulty with administration. ${ }^{2}$ In addition, dosing error rates range from $0.1 \%-20.7 \%$ in older adults who draw insulin from a vial. ${ }^{3}$

Advances in insulin delivery systems, namely the development of the insulin pen delivery systems (IPDS), can assist patients in overcoming their concerns. Research has demonstrated advantages to using IPDS compared to traditional vial and syringe. ${ }^{4-13}$ IPDS is associated with better perceived glycemic control, increased patient preference, increased adherence, fewer hypoglycemic events, reduced emergency room visits, and lower annual treatment costs. ${ }^{4-9}$ Multiple studies have shown that the majority of patients prefer to use an IPDS compared to the traditional vial and syringe due to convenience, ease of use, discreetness in public, and improved quality of life. ${ }^{4,10-12}$ Studies also show that adherence to insulin therapy improves with the use of pen devices compared to vial and syringe. . $^{5-10,13}$

Corresponding author: Stefanie P. Ferreri, UNC Eshelman School of Pharmacy, Beard Hall 115B, Campus Box 7574, Chapel Hill, NC 27599-7574, Phone: 919-843-9765, Fax: 919-843-3861, Email: stefanie ferreri@unc.edu
Despite these advantages, only $15-20 \%$ of patients in the United States use IPDS compared to Europe and Japan, where $66-90 \%$ of patients use IPDS. ${ }^{14-15}$ Several factors contribute to low usage, including cost and prescription drug coverage limitations. In addition, there may be lack of awareness of IPDS advantages by prescribers or patients. ${ }^{16}$

Community-based pharmacists can serve as a resource to educate prescribers and patients about optimal insulin delivery choices, such as IPDS. The Asheville Project and the Diabetes Ten City Challenge have demonstrated that community-based pharmacists can positively impact the outcomes of patients with diabetes. These studies have shown that when patients work with community pharmacists, biometric measures improve and healthcare costs decrease. ${ }^{17-}$

${ }^{22}$ At every follow-up visit during the Asheville Project, patients showed an improvement in A1C from baseline. ${ }^{17}$ The total direct medical costs decreased by more than $\$ 600$ per patient per year. ${ }^{17}$ The latest diabetes initiative by the APhA Foundation, Project IMPACT: Diabetes, also demonstrated that pharmacists can play a vital role in improving patient outcomes, with an average decrease of $0.8 \%$ in $\mathrm{A}_{1 \mathrm{C}}{ }^{22}$ In addition, patients enrolled in the Patient Self-Management Program for Diabetes reported they were very satisfied or satisfied with the diabetes care provided by pharmacists. ${ }^{20}$ However, there has been no published research specifically on the pharmacists' role in converting patients from vial and syringe to IPDS. The purpose of this study is to describe a vialto-pen conversion program driven by community-based pharmacists and determine conversion success rate. 


\section{Methods}

A retrospective analysis of a community-based pharmacist initiated vial-to-pen conversion program was conducted. The study was reviewed and approved by the Institutional Review Board (IRB) at the University of North Carolina at Chapel Hill.

Patients were included if they were at least 18 years of age, spoke English, and had at least one prescription for an insulin vial product (either rapid-acting, long-acting, or premixed insulin) and syringes. Patients were excluded if they had government or state-funded prescription insurance, or if they did not have a prescription for syringes to account for the possibility of insulin pump usage.

This conversion program took place within a regional chain pharmacy in North Carolina. This chain consisted of approximately 70 stores, which provided traditional and clinical pharmacy services. Some locations offered point-ofcare testing (A1C, blood glucose, and lipid panels) and an American Diabetes Association-recognized education program. At the time, the company had two classifications of pharmacist employees: clinical pharmacists and store pharmacists. Clinical pharmacists were residency trained or had at least 3 years of experience providing clinical pharmacy services, such as Medication Therapy Management (MTM), immunizations, and diabetes education. Clinical pharmacists were assigned to one specific store location but also provided these clinical services to other pharmacies in the company, where no clinical pharmacist resided. Store pharmacists performed daily prescription dispensing and attended to any additional healthcare needs for patients at their pharmacy. In the vial-to-pen conversion program, ten clinical pharmacists initiated the process and store pharmacists completed the conversion process. Prior to the start of the program, clinical pharmacists attended a webinar training them in program details.

Figure 1 depicts the step-by-step process by which patients were contacted and converted from vial-to-pen. To identify eligible patients, a report was generated by the prescription claims database, identifying 200 prescriptions filled for an insulin vial product and syringes. From November 2011 to June 2012, patients were contacted by clinical pharmacists and informed of the availability and potential benefits of IPDS. If the patient agreed to the switch, the clinical pharmacist contacted the prescriber to obtain a new prescription. New prescriptions were sent to the store pharmacist, who dispensed the pen and educated the patient on its use. Prescription refill records were tracked for six months postintervention to determine whether patients who were converted remained on the IPDS. Clinical pharmacists were required to document attempted contacts and conversion results in a spreadsheet.
A retrospective review of the program was conducted in fall of 2012. The primary endpoint was vial-to-pen conversion success rate, defined as number of prescriptions converted from vial-to-pen out of the number of prescriptions that could have been converted for those patients the clinical pharmacists reached. Secondary endpoints collected were the average number of attempts to contact a patient, patient willingness to switch, average number of attempts to contact prescriber (per prescription), and prescriber acceptance rate, defined as the number of prescriptions approved out of the number of recommended conversions. In addition, the insulin product used by the patient at least six months postconversion was obtained from the pharmacy's dispensing system to determine whether patients who were converted remained on the IPDS. This endpoint was based on the last product filled for the patient at one of the company's stores, even if they were no longer using the pharmacy. Results were analyzed using descriptive statistics.

\section{Results}

Figure 2 depicts the breakdown of patients included and excluded in the program. In all, $26 \%$ of prescriptions (32/121) were successfully converted from vial-to-pen, representing 30 patients who received an IPDS and were provided education on use of the device by a pharmacist. Of the 200 eligible prescriptions identified for conversion, 5 prescriptions were excluded due to patients using an insulin pump, no longer filling at the pharmacy, or not speaking English (according to dispensing system). Of the 195 remaining prescriptions, 74 prescriptions were not converted because the clinical pharmacist was unable to reach the patient due to incorrect or disconnected phone numbers or no response after 3 attempts. The average number of attempts to contact patients was 1.8. Of the patients contacted, 52\% (55/105) expressed a willingness to switch to a pen. Clinical pharmacists made an average of 1.3 attempts to contact prescribers by fax. Of the vial-to-pen conversion recommendations made to prescribers, $71 \%(46 / 65)$ were accepted. Of those not accepted, some were denied, but for most, the provider did not respond. In the end, 32 pen prescriptions were dispensed by the pharmacy; $30 \%$ $(14 / 46)$ of pen prescriptions approved by prescribers were not picked up by the patient for unknown reasons. These prescriptions were returned to stock and put "on hold" in the dispensing system. Of the prescriptions successfully converted to an IPDS, 84\% (27/32) were still dispensed as pen products six months following the conversion.

\section{Discussion}

This analysis demonstrates that a community-based pharmacist-driven vial-to-pen conversion program is feasible and pharmacists can influence the initiation of IPDS in patients using insulin vial and syringe. After a clinical pharmacist educated patients on the benefits of using an IPDS, 52\% of patients were interested in switching products. Literature confirms that endocrinologists influence pen initiation; 
however, to the authors' knowledge, this is the first report that documents community-based pharmacists serving in this role. ${ }^{23}$ Most prescribers were receptive of recommendations to convert patients from insulin vials to pens, with $71 \%$ of recommendations approved. This is more favorable than prescriber acceptance of pharmacists' drug therapy recommendations for patients with diabetes (60.2\%) and recommendations made during MTM services (47.2\%). ${ }^{24-25}$

It appears that both prescribers and patients have begun to recognize the advantages of IPDS over vial and syringe. While IPDS represent only $15-20 \%$ of the insulin delivery system market, patients newly initiated on insulin are more likely to be started on an IPDS than in the past. ${ }^{14-15,26}$ In $2008,48.5 \%$ of patients with type 2 diabetes who started on insulin used an IPDS compared to only $10.6 \%$ in $2004 .{ }^{26}$ Even with this IPDS usage increase by patients, the overall rate of usage remains low in the US.

\section{Limitations}

There were multiple limitations to both implementation and analysis of the program success. The largest obstacle for the clinical pharmacists was being able to reach each patient. An additional 74 prescriptions could have potentially been converted if the clinical pharmacist would have been able to contact the patient. Perhaps more patients could have been reached and converted if the intervention was driven by store pharmacists, who see the patients on a regular basis, and patients may have been more comfortable with this model. This would allow pharmacists to make the intervention in person, when the patients pick up prescriptions, rather than making phone calls that may or may not result in an intervention.

An additional limitation of this study is that some patients identified in the report generated from the dispensing system were not eligible for conversion and some patients who could have benefited from the switch may not have been identified, as syringes may be purchased over the counter in North Carolina. Clinical pharmacists were not asked to document patients' reasoning for not switching products, so these barriers remain unknown. Although $71 \%$ of pen prescription recommendations were approved by prescribers, $30 \%$ of the approved prescriptions were not picked up by the patient. Pharmacists were not required to document reasons for the patient not receiving the prescription. A possible explanation may be a higher cost for IPDS based on the patient's prescription insurance since the cost was not known until the prescription was received and processed. However, literature shows that while pharmacy costs are higher for pens compared to vials, annual healthcare costs are lower. ${ }^{8}$

In addition, this analysis examined success of the conversion program and patient and prescriber acceptance. It did not include analyses of cost-effectiveness or glucose control, which are vital components of successful insulin therapy.

While implementation of the vial-to-pen conversion program was successful in converting patients to optimal insulin products, a relatively small number of patients were affected given the size of the pharmacy company (30 patients, 32 prescriptions). Following the program described in this study, a company-wide vial-to-pen conversion program was implemented. This put the entire conversion process in the hands of store pharmacists who already had established relationships with their patients. This might be a more realistic model for other companies who wish to incorporate this type of service.

\section{Conclusions}

This study demonstrates that community-based pharmacists can increase use of IPDS in patients with diabetes. Communitybased pharmacists are in a position to educate patients on insulin delivery choices, identify patients who many benefit from switching products, and assist these patients with selection of the optimal product. In this role, communitybased pharmacists can help increase overall use of IPDS in the US.

Author Disclosure Statement: No competing financial interests exist.

Acknowledgements: none

Author Notes: During the time this study was conducted, Dr. Hannings was a PGY2 Community Pharmacy/Academia Resident with UNC Eshelman School of Pharmacy and Kerr Drug. Dr. Michaels and Dr. Hiller were employed by Kerr Drug at the time of the study.

Previous Presentation: This article describes the findings of an abstract presented at the American Pharmacists Association Annual Meeting March 2, 2013, Los Angeles, CA 


\section{References}

1. National diabetes statistics report, 2014: estimates of diabetes and its burden in the United States. Centers for Disease Control and Prevention website. http://www.cdc.gov/diabetes/pdfs/data/2014-reportestimates-of-diabetes-and-its-burden-in-the-unitedstates.pdf. Updated May 15, 2015. Accessed June 17, 2019.

2. Karter AJ, Subramanian U, Saha C, Crosson JC, Parker MM, Swain $\mathrm{BE}$, et al. Barriers to insulin initiation: the translating research into action for diabetes insulin starts project. Diabetes Care. 2010;33:733-5.

3. Coscelli C, Calabrese G, Pisu E, et al. Use of premixed insulin among the elderly. Diabetes Care. 1992;15(11):1628-30.

4. Rubin RR, Peyrot MP. Quality of life, treatment satisfaction, and treatment preference associated with use of a pen device delivering a premixed 70/30 insulin aspart suspension (aspart protamine suspension/soluble aspart) versus alternative treatment strategies. Diabetes Care. 2004;27(10):2495-97.

5. Lee WC, Balu S, Cobden D, Joshi AV, Pashos CL. Medication adherence and the associated health-economic impact among patients with type 2 diabetes mellitus converting to insulin pen therapy: an analysis of third-party managed care claims data. Clin Ther. 2006;28(10):1712-25.

6. Shelmet J, Schwartz S, Cappleman J, Peterson G, Skovlund $S$, Lytzen $L$, et al. Preference and resource utilization in elderly patients: InnoLet versus vial/syringe. Diabetes Res Clin Practice. 2004;63:27-35.

7. Pawaskar MD, Camacho FT, Anderson RT, Cobden D, Joshi AV, Balkrishnan R. Health care costs and medication adherence associated with initiation of insulin pen therapy in Medicaid-enrolled patients with type 2 diabetes: a retrospective database analysis. Clin Ther. 2007;29(6 Pt 1):1294-305.

8. Ayyagari R, Wei W, Cheng D, Pan C, Signorovitch J, Wu EQ. Effect of adherence and insulin delivery system on clinical and economic outcomes among patients with type 2 diabetes initiating insulin treatment. Value Health. 2015;18(2):198-205.

9. Lasalvia P, Barahona-Correa JE, Romero-Alvernia DM, et al. Pen devices for insulin self-administration compared with needle and vial: systematic review of the literature and meta-analysis. J Diabetes Sci Technol. 2016;10(4):959-66.

10. Graff MR, McClanahan MA. Assessment by patients with diabetes mellitus of two insulin pen delivery systems versus a vial and syringe. Clin Ther. 1998;20(3):486-96.

11. Molife C, Lee LJ, Shi L, Sawhney M, Lenox SM. Assessment of patient-reported outcomes of insulin pen devices versus conventional vial and syringe. Diabetes Technol \& Ther. 2009;11(8):529-538.

12. Korytkowski M, Bell D, Jacobsen C, Suwannasari R. A multicenter, randomized, open-label, comparative, twoperiod crossover trial of preference, efficacy, and safety profiles of a prefilled, disposable pen and conventional vial/syringe for insulin injection in patients with type 1 or 2 diabetes mellitus. Clin Ther. 2003; 25(11):2836-2848.
13. Xie L, Zhou S, Wei W, Gill J, Pan C, Baser O. Does pen help? A real-world outcomes study of switching from vial to disposable pen among insulin glargine-treated patients with type 2 diabetes mellitus. Diabetes Technol \& Ther. 2013;15(3):230-6.

14. The state of insulin injection. Diabetes Health website. https://www.diabeteshealth.com/the-state-of-insulininjection/. Updated September 11, 2011. Accessed June 17, 2019.

15. D'Arrigo T. Grudge Match: Pens vs. Syringes. Endocrine News website. https://endocrinenews.endocrine.org/april2014-grudge-match-pens-vs-syringes/. Updated April 2014. Accessed June 17, 2019.

16. Pearson TL: Practical aspects of insulin pen devices. J Diabetes Sci Technol. 2010;4(3):522-31.

17. Cranor CW, Christensen DB: The Asheville Project: shortterm outcomes of a community pharmacy diabetes care program. J Am Pharm Assoc. 2003;43:149-59.

18. Cranor CW, Christensen DB: The Asheville Project: factors associated with outcomes of a community pharmacy diabetes care program. J Am Pharm Assoc. 2003;43:16072.

19. Cranor CW, Bunting BA, Christensen DB: The Asheville Project: long-term clinical and economic outcomes in a community pharmacy diabetes care program. J Am Pharm Assoc. 2003;43:173-84.

20. Garrett DG, Bluml DB: Patient Self-Management Program for Diabetes: first-year clinical, humanistic, and economic outcomes. J Am Pharm Assoc. 2005;45:130-7.

21. Fera T, Bluml DB, Ellis WM: Diabetes Ten City Challenge: final economic and clinical results. J Am Pharm Assoc. 2009;49:e52-e60.

22. Bluml BM, Watson LL, Skelton JB, et al. Improving outcomes for diverse populations disproportionately affected by diabetes: final results of Project IMPACT: Diabetes. J Am Pharm Assoc. 2014 Sept-Oct;

23. 54:477-85. Peyrot $M$, Rubin RR: Physician perception and recommendations of insulin pens for patients with type 2 diabetes mellitus. Curr Med Res Opin. 2008;24(8):2413-22.

24. DeName B, Divine H, Nicholas A, Steinke DT, Johnson CL. Identification of medication-related problems and health care provider acceptance of pharmacist recommendations in the DiabetesCARE program. J Am Pharm Assoc. 2008;48:731-6.

25. Perera PN, Guy MC, Sweaney AM, Boesen KP. Evaluation of prescriber responses to pharmacist recommendations communicated by fax in a medication therapy management program (MTMP). J Manag Care Pharm. 2011 Jun;17(5):345-354.

26. Lee $L J, L i Q$, Reynolds MW, Engelman W. Trend analyses of insulin delivery systems in the United States. J Diabetes Sci Technol. 2011;5(5):1116-23. 
Figure 1: Vial-to-Pen Conversion Process

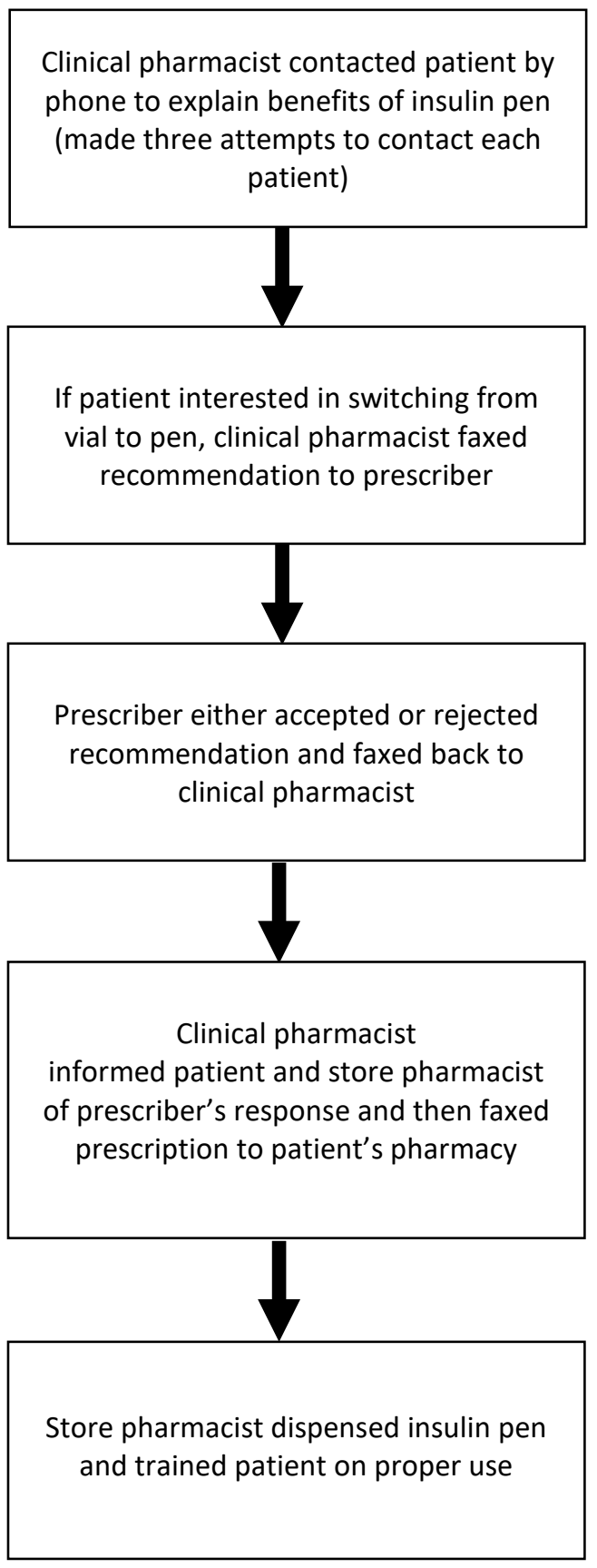


Figure 2: Flow Diagram of Patient Disposition in the Conversion Program

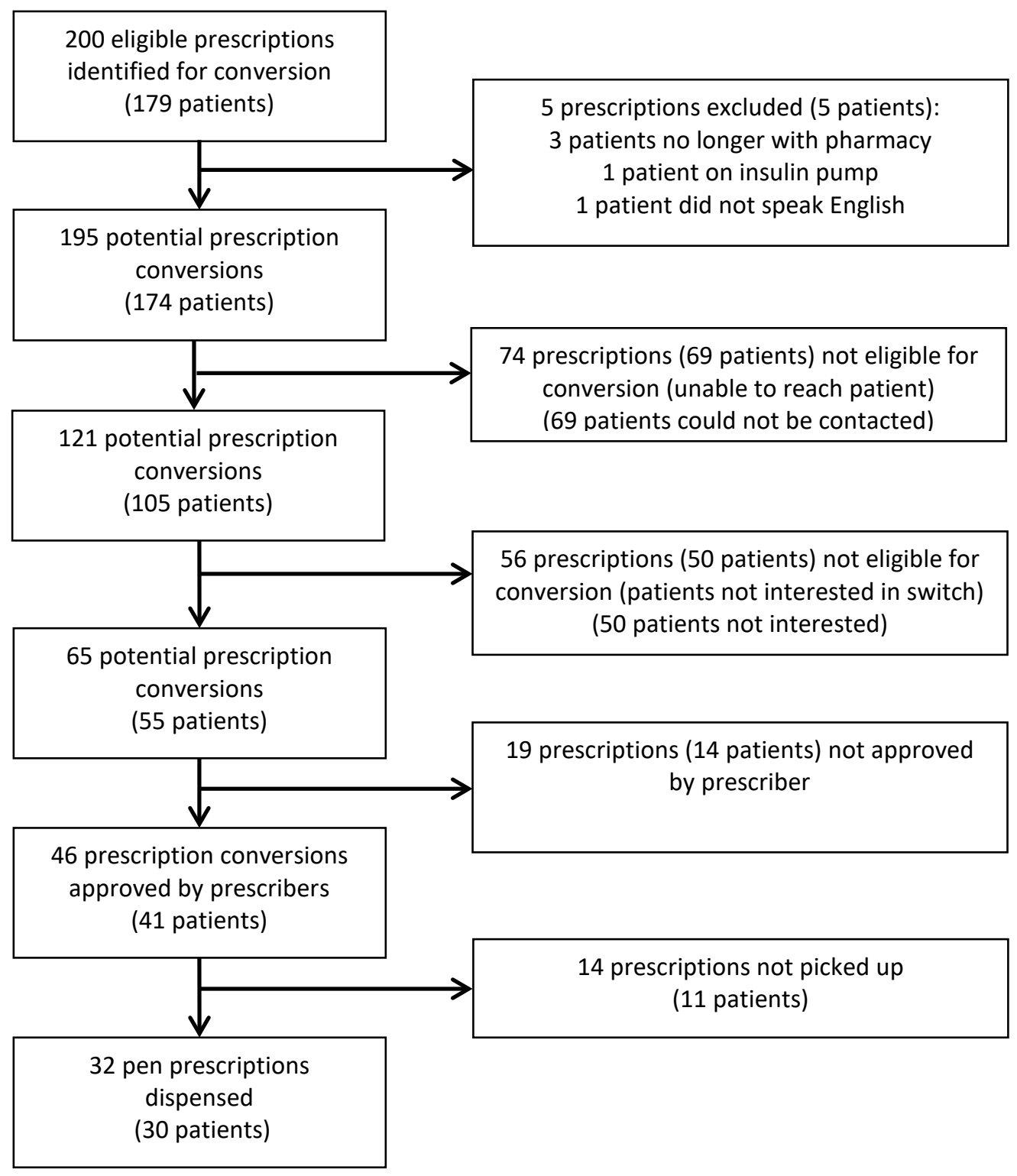

